\title{
Detection of subclinical autonomic neuropathy in constipated patients using a sweat test
}

\author{
D Altomare, M-A Pilot, M Scott, N Williams, M Rubino, L Ilincic, D Waldron
}

\begin{abstract}
Chronic idiopathic constipation may be the result of an autonomic neuropathy. This hypothesis was tested in 23 constipated patients and 17 age matched controls, using the acetylcholine sweat spot test devised to test autonomic integrity in diabetes. Acetylcholine (0.01\%) was injected in the dorsum of the foot painted with a mixture of starch and iodine. Active sweat glands appeared on the surface of the skin as small black dots which were photographed and counted, using a grid with 60 subareas. Two measurements were made: the number of dots per unit subarea (sweat spot test score) and the \% number of abnormal subareas (with less than six spots). These two parameters were correlated. The median sweat spot test score was 9.53 in patients and 13.92 in controls $(p=0.0001)$, the receiver operating characteristic curve showing that a score of 12 delimited normal and abnormal subjects. Increasing age was correlated with a low score in patients, probably because of prolonged symptoms. Seventy per cent of patients and one control had a borderline or abnormal number of subareas. These results suggest that idiopathic constipation is associated with a degree of autonomic denervation. The sweat spot test is an easy, inexpensive method to test this hypothesis and deserves a place in the clinical assessment of slow transit constipated patients.

(Gut 1992; 33: 1539-1543)
\end{abstract}

An intact autonomic nervous supply to the bowel is essential for normal function. Damage to one or both branches of the autonomic nervous system as a result of trauma, neoplasia, diabetes, infectious diseases, or neuronal degenerative diseases frequently leads to various gut motility (and secretory) problems, the most common being chronic constipation.

For many patients with severe chronic constipation no cause can be found, hence the condition is labelled as idiopathic. Chronic idiopathic constipation, however, is often associated with other motility disturbances involving multiple organs, suggesting an underlying systemic disease of the autonomic nervous system. ${ }^{1-3}$ Previous attempts to detect an early autonomic neuropathy in these patients, based on the conventional cardiovascular tests suggested by Ewing and Clark, ${ }^{+}$have given inconsistent results, ${ }^{5}$ probably because of their poor sensitivity and specificity. Sympathetic denervation has also been assessed by investigating sweating ability in patients with unexplained gut dysmotility using the thermoregulatory sweat test and quantitative sudomotor axon reflex test. ${ }^{6}$ These tests require complex procedures, however, and specialised equipment which are not always readily available. A new test of autonomic integrity, the acetylcholine sweat spot test, ${ }^{7}$ has recently been claimed to be more sensitive than conventional tests, and reliable for quantification of the severity of autonomic nervous system damage.

The aim of this study is to investigate whether a subclinical impairment of the autonomic nervous system is present in patients with slow transit idiopathic constipation, and whether it has a possible role in the pathogenesis of this motility disorder.

\section{Methods}

PATIENTS

Twenty three patients ( 21 women, median age 42 years, range 28 to 78 ) with severe chronic idiopathic constipation entered the study. Severe chronic constipation was defined as constipation without bowel obstruction, lasting for more than 18 months, not responding to high fibre diets, with less than two bowel movements per week and without an alternating bowel habit (diarrhoea and constipation). Each patient had a proctometrogram to exclude Hirshsprung's disease and an integrated dynamic defecography ${ }^{8}$ to identify anismus or other relevant anorectal abnormalities. A radioopaque marker test showed that all patients had delayed colonic transit, which was confirmed in 11 patients by a radioisotope transit study. ${ }^{9}$ Nine patients had a partial or total colectomy for constipation before this study.

\section{CONTROLS}

Seventeen age matched healthy volunteers (12 women, median age 42 years, range 28-69) served as controls. The exclusion criteria for the selection of controls were constipation or other gastrointestinal problems.

The acetylcholine sweat spot test was carried out injecting $0.1 \mathrm{ml}$ of a $1 \%$ solution of acetylcholine (Miochol - CooperVision Ltd, Southampton, UK) intradermally using a 25 gauge needle in a marked area of the dorsum of the foot which had previously been painted with a $2 \%$ alcholic iodine solution and a suspension of maize starch in arachis oil $(100 \mathrm{~g}$ in $100 \mathrm{ml})$. The reaction between sweat, iodine, and starch could be seen about four to five minutes later as fine black dots corresponding to functioning sweat glands (Fig 1). The marked skin area was photographed and the number of dots counted 


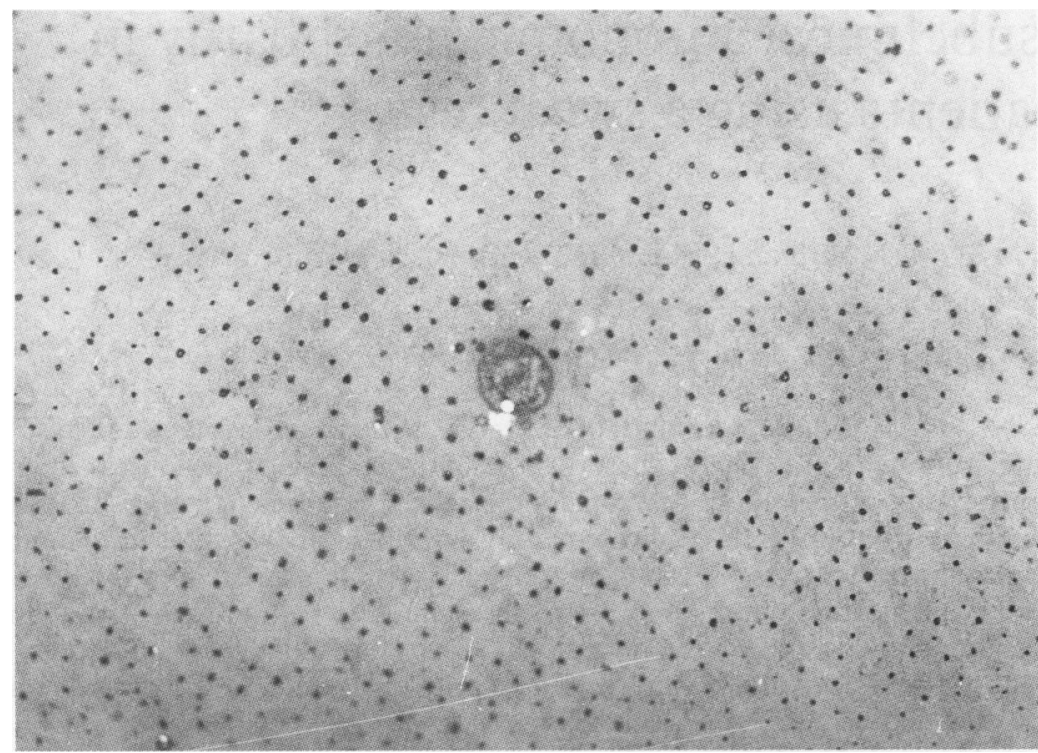

Figure 1: Photograph of acetyl choline stimulated sweat glands in the dorsum of the foot painted with a maize starch/iodine solution. The artifact in the middle is the point of injection. Control subject, sweat spot test score 16.00. Note the even distribution of glands. a screen using a $20 \times 20 \mathrm{~cm}$ grid (64 subareas) centred on the injection site.

The number of dots included in each $2.5 \times 2.5$ $\mathrm{cm}$ subarea of the grid were counted, excluding the four central subareas around the site of injection. The dots touching the upper and left side borders of each subarea were counted while those on the lower and right side were ignored. For 21 subjects ( 16 patients and five controls) the count was made at different times by three independent observers, two of whom were

Figure 2: Sweat spot test response (SST score, number of sweat glands per unit area) in 23 constipated patients (closed circles) and 17 controls (open circles). The short horizontal line is the median in each group, $p<0 \cdot 0001$, Mann-Whitney $U$ test.

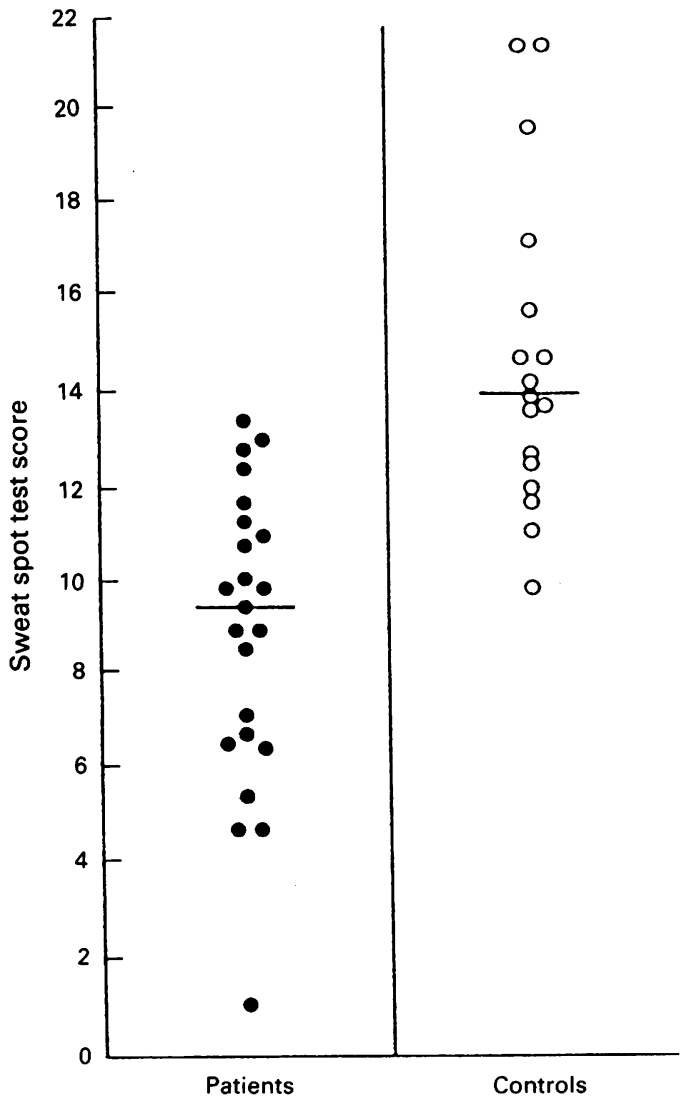

unaware of the diagnosis. The final value obtained for statistical analysis corresponded to the median of the three observed values for each patient. The test took about 10 minutes to complete and counting the dots on the photo required 10 to 20 minutes.

As suggested by Ryder and coworkers ${ }^{7}$ the occurrence of five $(8.3 \%)$ or more subareas with less than six dots was chosen as the criterion for abnormality. The mean number of dots/subarea (sweat spot test score) was also recorded for each subject.

\section{STATISTICAL ANALYSIS}

The Mann-Whitney $U$ test was used in the comparison of the sweat spot test scores between patients and controls, and Fisher's exact test for the analysis of abnormal subareas. The effect of a confounding factor such as age, and the relationship between the number of abnormal subareas and the sweat spot test score were analysed using the Spearman correlation coefficient (rho) with correction for ties.

The reliability of the method was also investigated by measuring the level of agreement between observers using the K-statistic. The subjects were allotted to three arbitrarily defined categories for both the sweat spot test score $(>11,11-9.5,<9.5)$ and the percentage number of abnormal subareas $(0,0-0.033,>0.033)$. This was believed necessary, because random agreement between three observers for only two categories - that is, normal/abnormal - is very likely to occur, making results difficult to evaluate.

The sensitivity, specificity, and efficiency of the sweat spot test were calculated for both the sweat spot test score and the percentage number of abnormal subareas, and receiver operating characteristic curves were drawn for both parameters.

\section{Results}

The median sweat spot test score in patients $(9.53$, range 1.20 to 13.4$)$ was significantly lower than in controls (median 13.92, range 9.95 to $21 \cdot 5, p=0.0001$, Fig 2). In controls the distribution of dots on the skin surface appeared to be uniform (Fig 1) whereas patients had a more patchy distribution (Fig 3).

There was no significant difference in age between the group of 23 patients (median age 42, range 28-78) and the 17 controls (median age 42 , range 28-69). In the control group there was no correlation between age and the sweat spot test score while in patients there was a moderate but significant negative correlation, older patients having a lower sweat spot test score ( $\mathrm{rho}=0.45, \mathrm{p}=\mathbf{0} .035$, Spearman correlation coefficient).

The number of abnormal subareas - that is, those subareas containing fewer than six dots were also analysed for differences between the two groups. Because of blurring of the photographic image caused by focusing difficulties in some subjects, the number of subareas where counting was possible was less than the maximum of 60 . In order to compare the number of 


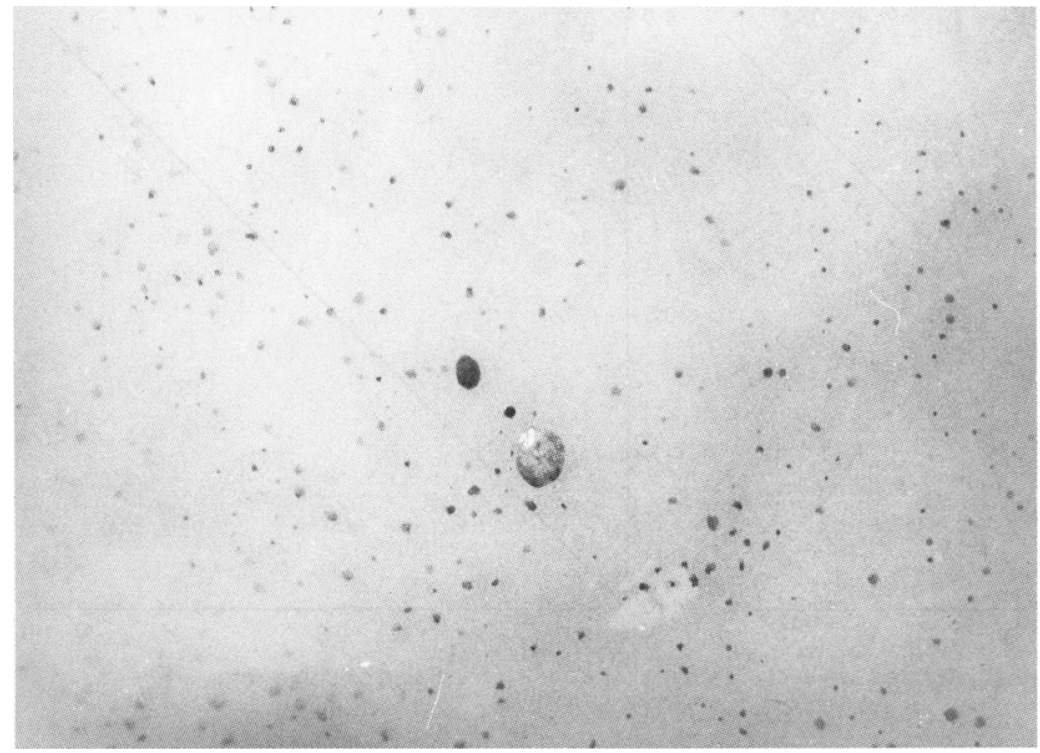

Figure 3: Sweat response to acetylcholine in a patient with a sweat spot test score of $1 \cdot 20$. There are few active glands unevenly distributed.

abnormal subareas observed in the two groups the results were normalised. According to Ryder's classification, the presence of abnormal subareas of 0.083 (five of 60 ) or higher was considered to show a degree of autonomic impairment. A result of two to four abnormal subareas $(0.033-0.067)$ were defined as being borderline. Eleven of 23 patients and none of the controls had more than 0.083 abnormal subareas $(\mathrm{p}<0 \cdot 001$, Fisher's exact test). Five patients and one control fell into the borderline category.

The Spearman correlation test showed a significant correlation $(p=0.0001$, rho $=-0.85)$ between the number of abnormal subareas and the sweat spot test score for all subjects (Fig 4).

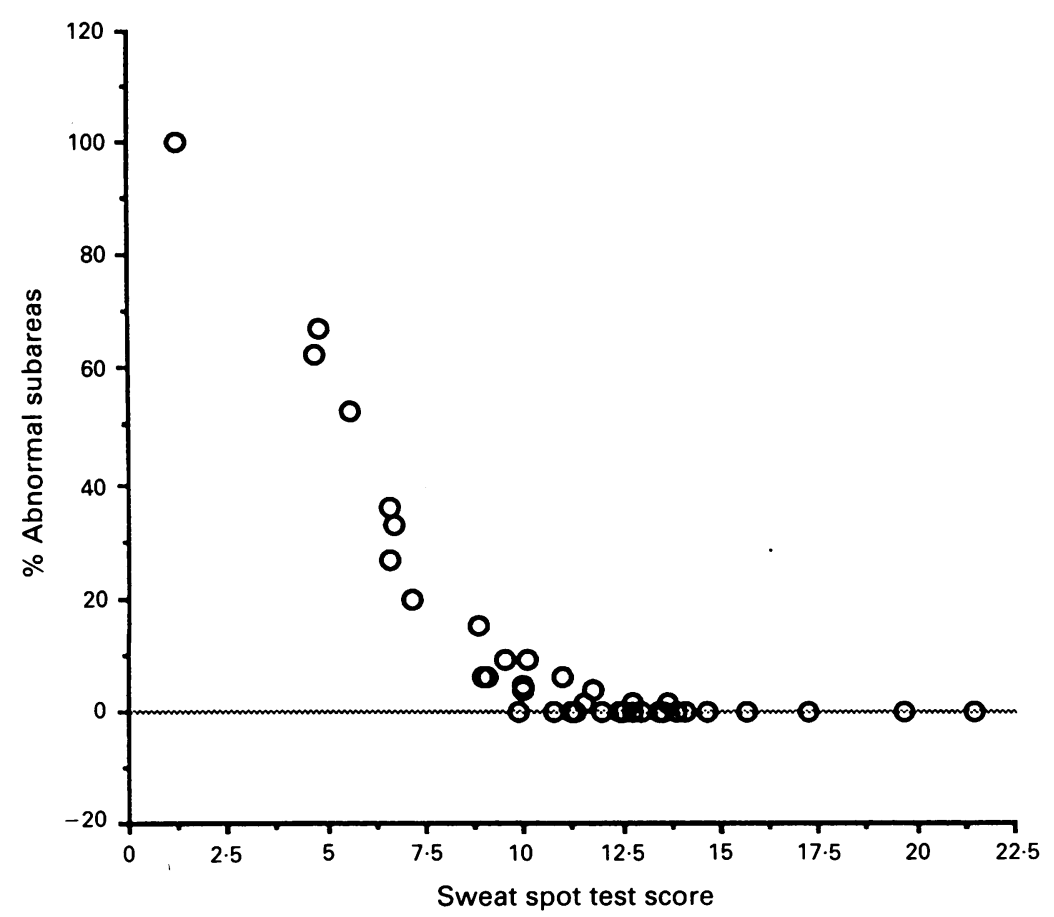

Figure 4: Scattergram of the relationship between the sweat spot test score and the percentage number of abnormal subareas. The Spearman correlation coefficient (rho) was $-0 \cdot 85$, $p=0.0001$.
When the patients were analysed as a separate group, the correlation between the sweat spot test score and the \% number of abnormal subareas was also significant $(\mathrm{p}=0.0001$, rho $=$ $-0.91)$.

In the 21 subjects in whom the number of sweat glands were counted by three independent observers, the correlation between their observations were assessed. The $K$ value was 0.81 $(z=11 \cdot 83, p<0.0001)$ for the agreement on the sweat spot test score and $0.78 \quad(z=10.65$, $\mathrm{p}<0.0001)$ for the agreement on the abnormal subareas.

The receiver operating characteristic curve is illustrated in Figure 5. The highest efficiency $(83 \%)$ occurs with a sweat spot test score of 12 for the demarcation of normal from abnormal subjects (sensitivity 0.83 , specificity 0.82 ). For the abnormal subareas the highest efficiency was with a percentage of abnormal areas of 0.033 or more (sensitivity $0 \cdot 65$, specificity $1 \cdot 0$ ).

\section{Discussion}

The aetiology of chronic idiopathic constipation is uncertain, and attempts to cure it have given poor results. Surgical treatment of severe chronic constipation not responding to medication had an inconsistent and unsatisfactory outcome, resulting sometimes in intractable diarrhoea requiring a definitive terminal ileostomy or in persistence of abdominal pain, bloating, and discomfort. ${ }^{1011}$

The mechanisms underlying constipation have been investigated. Involvement of endogenous opioids and their receptors in the regulation of colonic transit was proposed by Kreek ${ }^{12}$ but further investigation ${ }^{13}$ failed to confirm this. The observation that most of the patients with chronic idiopathic constipation, like the population in our study, are women, has suggested the possible role of a disturbed hormonal environment in the pathogenesis ${ }^{1+}$ but these findings have not been substantiated. ${ }^{15}$ Psychological factors have also been indicated as a possible cause for some cases of idiopathic constipation, ${ }^{16}$ but there is no strong evidence to support this. Degeneration of intrinsic enteric neurones has also been suggested as a possible cause, ${ }^{12}$ but it might be the secondary result of long lasting bowel distension and laxative abuse.

Impairment of the autonomic nervous system was proposed as a cause of bowel motility dysfunction as early as $1928 .{ }^{17}$ The observation that constipation is frequently associated with other subclinical or clinically evident motility disorders of the bladder, oesophagus, ileum ${ }^{78} 19$ and that it is a common bowel disturbance in diseases involving the autonomic nervous system suggests the existence of a relationship between autonomic neuropathy and constipation. An early neuropathy may be present without major disturbances such as orthostatic hypotension, impotence or urinary incontinence, but it may be enough to disturb the fine regulation of gut motility, causing slow transit constipation. The cardiovascular tests ${ }^{4}$ conventionally used for detecting sympathetic autonomic neuropathy may not be adequate to detect a mild, otherwise asymptomatic neuropathy. ${ }^{20}$ 
Figure 5: Receiver operating characteristic curves for the sweat spot test score $(A)$ and the percentage number of abnormal subareas $(B)$.
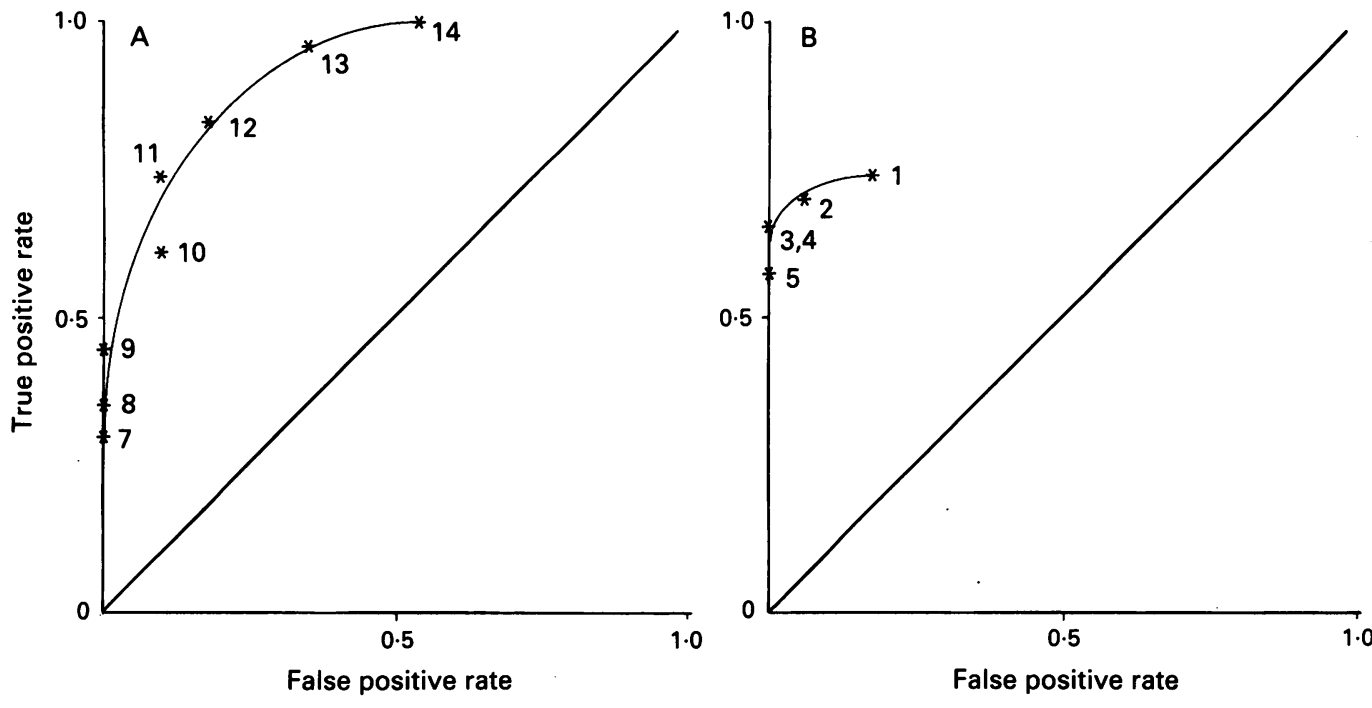

Impairment of sweating is associated with various manifestations of autonomic neuropathy with and without the presence of other symptoms such as postural hypotension. ${ }^{21}$ Sweat regulation has been used as a test of autonomic nervous system function in diabetics since $1942 .{ }^{22}$ Camilleri and Fealey, ${ }^{6}$ using the thermoregulatory test and a quantitative sudomotor axon reflex test, showed in eight patients with gastrointestinal problems that an idiopathic autonomic denervation may be an aetiological factor in non-organic intestinal dysmotility. All these patients had patchy anhidrosis and two had positive cardiovascular tests.

\section{INTERPRETATION OF THE SWEAT SPOT TEST RESPONSE}

The intradermal injection of acetylcholine is effective in stimulating sweat glands because acetylcholine, and not noradrenaline, is the post ganglionic sympathetic neurotransmitter in sudomotor fibres. ${ }^{23}$ In the presence of exogenous acetylcholine a normal sweat response should therefore be expected even if nerve degeneration precludes adequate transmitter release. Normal response to the test, however, still depends on an intact nerve supply. Acetylcholine is known to be cotransmitted with vasoactive intestinal polypeptide at the synapse between the sympathetic nerve and the sweat gland, vasoactive intestinal polypeptide probably being responsible for the local vasodilation which is necessary for a normal sweat response. ${ }^{21}{ }^{24} \mathrm{We}$ suggest that the presence of acetylcholine alone will not provide an adequate stimulus for the sweat response in the event of nerve degeneration, as the full response requires the combined action of both transmitters, provided that the receptors remain intact after nerve degeneration.

There is no evidence to suggest that sweat glands and their receptors remain unchanged after nerve degeneration. In fact, the sweating response to acetylcholine is reported to diminish gradually (one to two years) after denervation. ${ }^{22}$ It is, therefore, possible that in the presence of an autonomic neuropathy of long duration the number of working sweat glands decreases and their distribution becomes irregular. In the original sweat spot test ${ }^{7}$ the foot was chosen because, impairment being more likely to occur in longer nerve fibres, the lower extremities would be the first to be involved in diabetic autonomic neuropathy. Although this theory has not been tested in other forms of autonomic neuropathy, we have assumed that the same holds true in cases where constipation was associated to a degree of autonomic neuropathy.

The sensitivity of the sweat spot test derived from the receiver operating characteristic curve is rather low at $83 \%$. The most likely explanation is that these constipated patients are a heterogeneous group including patients without autonomic nervous system dysfunction, whose loss of peristaltic activity might instead be the result of intrinsic nerve damage or other motility disorder. A score of 12 on the sweat spot test response appears to be the delimiting number between normal and abnormal subjects in this population. The curve for abnormal subareas has a limited sensitivity, but the specificity is high at 1 , for a scoring of $3 \%$ of abnormal subareas, which is the demarcation set by $\operatorname{Ryder}^{7}$ as a departure from normality.

This study suggests some degree of idiopathic autonomic denervation in patients with slow transit constipation. As with Ryder's findings in diabetics with autonomic neuropathy, ${ }^{7}$ most patients with slow transit constipation had a patchy anhidrosis and a number of working glands significantly below normal controls. In these patients, even when the number of glands was normal, their distribution was not uniform.

The significant decrease in sweat spot test score with increasing age would indicate that age is an influential factor in the response to the sweat spot test. There is a reported reduction both in the number of active sweat glands and in the secretory output of each gland with increasing age, ${ }^{25}$ a phenomenon which is not the result of a failure of the autonomic control of sweating but partly to trophic changes which occur with age in human skin. ${ }^{26}$ The correlation observed between age and loss of sweating ability, however, is only significant in subjects who are more than 70 years old, ${ }^{26}$ and only one of our patients 
was over 70. In the present study, controls were matched for age with the patient group, and as with Ryder's normal subjects, ${ }^{7}$ no relationship was shown between age and sweat spot test score in the control group. The decrease in spot density with age observed in patients with constipation is not therefore an age related factor, but is most probably related to the duration of the symptoms as most had been constipated for many years.

The loss of sudomotor function seen in constipated patients in this study would at first indicate a degree of impairment of the sympathetic nervous system which would result in the loss of inhibitory tone on gut smooth muscle. This would consequently lead to increased motility because parasympathetic activity would be unopposed by diminished sympathetic activity. Increased motility does not necessarily result in increased propulsion, however. Constipating drugs such as opioids increase motility while inhibiting propulsive activity, ${ }^{18}$ and abnormal hypermotility patterns have been reported to occur in the irritable bowel syndrome ${ }^{27}$ and constipation. ${ }^{19}$ We can speculate that increased excitability of the smooth muscle in the bowel because of failure of the inhibitory sympathetic modulation of parasympathetic activity could result in increased mixing and segmental activity of the colon with impaired coordination of peristaltic activity.

If an autonomic neuropathy is present, it is unlikely that a selective sympathetic impairment would occur in the presence of normal parasympathetic function. ${ }^{28}$ The decreased sweating function and slow transit constipation seen in our patients could be a consequence of a mild cholinergic dysautonomia, rather than a sign of a more prominent sympathetic failure. Several reports of extreme cases of selective cholinergic dysautonomia identify ileus (or constipation) and loss of sweating as two of the symptoms experienced by those patients..$^{29-31}$

The extreme complexity of the enteric nervous system with its non-adrenergic non-cholinergic neurones and its connections with the autonomic nervous system and higher neural centres precludes a simple explanation for the effects of autonomic neuropathy in constipation.

In conclusion, idiopathic constipation may well be an early manifestation of an otherwise asymptomatic systemic neuropathy. The acetylcholine sweat spot test is an easy, inexpensive, time saving test for detecting autonomic neuropathy. It is reliable for the quantification of the severity of nerve damage and deserves a place in routine clinical assessment of slow transit constipated patients.

The authors wish to thank Dr Robert Ryder for kindly showing us the intricacies of the sweat spot test, Dr Julian Roberts for providing data on intestinal transit time, and Dr Tom Koeze for help with the data on intest
statistics.
1 Feldman M, Schiller LR. Disorders of gastrointestinal motility associated with diabetes mellitus. Ann Intern Med 1983; 98: 378-84.

2 Kerrigan DD, Lucas MG, Sun WM, Donnelly TC, Read NW. Idiopathic constipation associated with impaired urethrovescical and sacral reflex function. $B r F$ Surg 1989; 76: 748-51.

3 Watier A, Devroede G, Duranceau A, Abdel-Rahman M, Duguay C, Forand MD, et al. Constipation with colonic inertia. A manifestation of systemic disease? Dig Dis $\mathrm{Sci}$ 1983; 28: 1025-33.

4 Ewing DJ, Clarke BF. Diagnosis and management of diabetic autonomic neuropathy. $\mathrm{BrMed}$ f 1982; 285: 916-8.

5 Waldron DJ, Williams NS, Kumar D, Hallan RI, Swash M. Is intractable constipation associated with a systemic autonomic neuropathy? [Abstract]. Br F Surg 1989; 76: 645 .

6 Camilleri M, Fealey RD. Idiopathic autonomic denervation in eight patients presenting with functional gastrointestinal eight patients presenting with functional gastrointestinal

7 Ryder REJ, Marshall R, Johnson KJ, Ryder AP, Owens DR, Hayes TM. Acetylcholine sweatspot test for autonomic denervation. Lancet 1988; i: 1303-5.

8 Womack NR, Williams NS, Holmfield JHM, Morrison JFB, Simpkins KC. New method for the dynamic assessment of anorectal function in constipation. $\mathrm{Br} \mathcal{F}$ Surg 1985; 72: 994-8.

9 Roberts JP, Waldron DW, Newell MA, Garvie NW, Williams NS. Comparison of radioopaque markers and radioisotopic assessment of whole bowel transit time in constipated patients. Gut 1990; 31: A617.

$10 \mathrm{Kamm}$ MA. The surgical treamtent of severe idiopathic constipation. Int 7 Colorect Dis 1987; 2: 229-35.

11 Leon SH, Krishnamurti S, Schuffler MD. Subtotal colectomy for severe idiopathic constipation: a follow-up study of 13 patients. Dig Dis Sci 1987; 32: 1249-54.

12 Kreek KJ, Schaefer RA, Hahn EF, Fishman J. Nalozone, a specific opioid antagonist, reverses chronic idiopathic constipation. Lancet 1983; i: 261-2

13 Fotherby KJ, Hunter JO. Idiopathic slow-transit constipation: whole gut transit times measured by a new simplified method, are not shortened by opioid antagonists. Aliment Pharmacol Ther 1987; 1: 331-8.

14 Preston DM, Lennard-Jones JE. Severe chronic constipation in young women: idiopathic slow transit constipation. Gut $1986 ; 27: 41-8$

15 Preston DM, Rees LH, Lennard Jones JE. Gynaecological disorders and hyperprolactinaemia in chronic constipation. Gut 1988; 24: A480.

16 Wald A, Hinds JP, Caruana BJ. Psychological and physiological characteristics of patients with severe idiopathic logical characteristics of patients with seve
constipation. Gastroenterology 1989; 97: 932-7.

17 Bockus HL, Bank J, Wilkinson SA. Neurogenic mucous colitis. Am F Med Sci 1928; 176: 813-28.

18 Painter SN, Truelove SC. The intraluminal pressure patterns in diverticulosis of the colon. Part II: The effect of morphine. Gut 1964; 5: 201-13.

19 Kumar D, Waldron D, Williams NS, Wingate DL. Slow transit constipation: a pan-enteric motor disorder? Gastroenterology 1989; 96: A277.

20 Fisher BM, Frier BM. Usefulness of cardiovascular tests of autonomic function in asymptomatic diabetic patients. Diabetes Res Clin Pract 1989; 6: 157-60.

21 Collins KJ. Autonomic control of sweat glands and disorders of sweating. In: Bannister R, ed. Autonomic failure. Oxford: of sweating. In: Bannister R, ed. Autonomi
Oxford University Press, 1988: 748-65.

22 Kahn D, Rothman S. Sweat response to acetylcholine. f Dermatol Invest 1942; 5: 431-44.

23 Dale HH, Feldberg W. The chemical transmission of secretory impulses to the sweat glands of the cat. $\mathcal{F}$ Physiol 1934; 82: 121-8

24 Lundberg JM, Hökfelt T, Schultsberg M, Uvnäs-Wallenstein $\mathrm{K}$, Köhler C, Said SJ. Occurrence of vasoactive intestinal polypeptide (VIP)-like immunoreactivity in certain cholinergic neurons of the cat: evidence from combined immunochemistry and acetylcholinesterase staining. Neuroscience 1979; 4: 1539-58.

25 Foster KG, Ellis FP, Doré C, Exton-Smith AN, Weiner JS. Sweat responses in the aged. Age Ageing 1976; 5: 91-101.

26 Collins KJ, Exton-Smith AN, James MH, Oliver DJ Functional changes in autonomic nervous responses with Functional changes in autonomic

27 Kumar D, Wingate DL. The irritable bowel syndrome: paroxysmal motor abnormalities. Lancet 1985; ii: 973-7.

28 Khurana RK. Acute and subacute autonomic neuropathies. In: Bannister R. Autonomic failure. Oxford: Oxford University Press, 1988: 624-31.

29 Vassallo M, Camilleri M, Caron L, Low P. Gastrointestina motor dysfunction in acquired selective cholinergic dysatonomia associated with infectious mononucleosis. Gastroenterology 1991; 100: 252-8.

30 Inamdar S, Easton LB, Lester G. Acquired postganglionic dysautonomia: Case report and review of the literature. Pediatrics 1982; 70: 976-8.

31 Young RR, Ashbury AK, Adams RD, Corbett JL. Pure pandysautonomia with recovery. Trans Am Neurol Assoc 1969; 94 : 355-7. 